\title{
Vacinação contra hepatite B em pessoas que vivem com HIV no Estado do Piauí,
}

\section{Brasil}

\author{
Hepatitis B vaccination in people living with HIV in the State of Piauí, Brazil \\ Vacunación contra la hepatitis B en personas que viven con el VIH en el Estado de Piauí, Brasil
}

Recebido: 22/12/2020 | Revisado: 30/12/2020 | Aceito: 07/05/2021 | Publicado: 21/05/2021

Evaldo Hipólito de Oliveira

ORCID: https://orcid.org/0000-0003-4180-012X Universidade Federal do Piauí, Brasil

E-mail: evaldohipolito@gmail.com

Anna Clara dos Santos Silva

ORCID: https://orcid.org/0000-0003-2873-1828

Universidade Federal do Piauí, Brasil

E-mail: annalidias@hotmail.com

Ian Vieira Rêgo

ORCID: https://orcid.org/0000-0001-7740-4714

Universidade Federal do Piauí, Brasil

E-mail: ianvieira9@hotmail.com

Talissa Brenda de Castro Lopes

ORCID: https://orcid.org/0000-0001-7694-1067

Universidade Federal do Piauí, Brasil

E-mail: talissa.brenda@hotmail.com

Karla Genini Lira Rocha

ORCID: https://orcid.org/0000-0002-1999-598X

Universidade Federal do Piauí, Brasil

E-mail: karlagenini@gmail.com

Leandro Oliveira Guimarães

ORCID: https://orcid.org/0000-0002-7038-0390

Universidade Federal do Piaú, Brasil

E-mail: leandroguimaraes99@yahoo.com.br

Albertina Costa Oliveira

ORCID: https://orcid.org/0000-0001-7153-1982

Centro Universitário Uninovafapi, Brasil

E-mail: betina.olliveira@gmail.com

Marcos Meneses de Oliveira

ORCID: https://orcid.org/0000-0001-7214-2030

Universidade Federal do Piauí, Brasil

E-mail: marcosmenzesoliver@gmail.com

Yasmim de Sousa Moura

ORCID: https://orcid.org/0000-0002-6868-8441

Universidade Federal do Piaú, Brasil

E-mail: yasmimmarks_19@hotmail.com

\begin{abstract}
Resumo
A hepatite B (HB) é responsável por diversos agravos incluindo complicações evolutivas como a cirrose e o carcinoma hepatocelular. A prevenção por meio da vacinação é essencial para pessoas que vivem com HIV, pois esse grupo apresenta maior taxa de progressão para a cronicidade da doença. O objetivo do trabalho foi analisar a cobertura vacinal contra o vírus da HB no estado do Piaú, por meio de um estudo soro epidemiológico de pacientes soropositivos cadastrados no Sistema de Controle de Exames Laboratoriais (SISCEL) do Laboratório Central de Saúde Pública do Piauí no ano de 2010. Foram analisados os dados de 569 pessoas HIV positivas, sendo escolhidas variáveis epidemiológicas como: idade, cor, escolaridade e marcadores sorológicos para hepatite B. Foi realizada ainda a determinação do marcador viral para hepatite B, o anti-HBs. Os achados foram filtrados e organizados para comparar as frequências de títulos de anti-HBs entre os indivíduos de sorologia positiva para HIV. Os valores de soroconversão para o anti-HBs foram observados em apenas 39,7\% dos participantes, o que confirma que ocorrem menores taxas da produção desses anticorpos em pacientes imunocomprometidos pelo HIV. De acordo com os títulos de anti-HBs, foram observados comportamentos-chave de vulnerabilidade que torna os pacientes suscetíveis à infecção, destacando-se: a renda familiar e escolaridade, que apontam para a necessidade da implementação de políticas públicas que atendam a população menos favorecida.
\end{abstract}

Palavras-chave: Imunização; Hepatite B; HIV; AIDS. 


\begin{abstract}
Hepatitis B (HB) is responsible for several conditions such as cirrhosis and hepatocellular carcinoma. Prevention through vaccination is essential for people living with HIV, since this group has a higher rate of progression to the chronicity of the disease. The objective of the study was to analyze vaccination coverage against HB virus in the state of Piauí, through an epidemiological serum study of seropositive patients registered in the Laboratory Examination Control System (SISCEL) of the Central Laboratory of Public Health of Piauí in the year 2010. Data from 569 HIVpositive people were analyzed, with epidemiological variables such as age, color, education and serological markers for hepatitis B. The viral marker for hepatitis B, anti-HBs, was also determined. The findings were filtered and organized to compare the frequencies of anti-HBs titers among HIV-positive individuals. Seroconversion values for anti-HBs were observed in only $39.7 \%$ of the participants, which confirms that lower rates of production of these antibodies occur in patients immunocompromised by HIV. According to the anti-HBs titles, key vulnerability behaviors were observed that make patients susceptible to infection, with emphasis on: family income and education, which point to the need to implement public policies that serve the population less favored.
\end{abstract}

Keywords: Immunization; Hepatitis B; HIV; AIDS.

\title{
Resumen
}

La hepatitis B (HB) es responsable de varias afecciones que incluyen complicaciones evolutivas como cirrosis y carcinoma hepatocelular. La prevención mediante la vacunación es fundamental para las personas que viven con el VIH, ya que este grupo tiene una mayor tasa de progresión a la cronicidad de la enfermedad. El objetivo del estudio fue analizar la cobertura de vacunación contra el virus HB en el estado de Piauí, a través de un estudio epidemiológico en suero de pacientes seropositivos registrados en el Sistema de Control de Examen de Laboratorio (SISCEL) del Laboratorio Central de Salud Pública de Piauí en el año 2010 Se analizaron datos de 569 personas VIH positivas, eligiendo variables epidemiológicas como edad, color, educación y marcadores serológicos de hepatitis B. También se determinó el marcador viral de hepatitis B, anti-HBs. Los hallazgos se filtraron y organizaron para comparar las frecuencias de títulos de anti-HBs entre individuos VIH positivos. Se observaron valores de seroconversión para antiHBs en solo el 39,7\% de los participantes, lo que confirma que las tasas más bajas de producción de estos anticuerpos ocurren en pacientes inmunodeprimidos por el VIH. De acuerdo con los títulos anti-HBs, se observaron conductas clave de vulnerabilidad que hacen que los pacientes sean susceptibles de contagio, con énfasis en: ingreso familiar y educación, que apuntan a la necesidad de implementar políticas públicas que atiendan a la población menos favorecida. Palabras clave: Inmunización; Hepatitis B; VIH; SIDA.

\section{Introdução}

As hepatites virais foram responsáveis por 1,34 milhão de mortes apenas no de ano 2015, um número comparável às mortes causadas por tuberculose e superior às causadas pelo HIV (World Health Organization, 2017). Dentre as hepatites, destaca-se a infecção hepática potencialmente fatal causada pelo vírus da hepatite B (HBV). Considerada um grande problema de saúde global (Davitkov \& Falck-Ytter, 2018; Organização Mundial da Saúde, 2019).

A transmissão do HBV ocorre pelo contato com sangue ou outros fluidos corporais, pelas vias parenterais (através do compartilhamento de agulhas, seringas, material de manicure e pedicure, lâminas de barbear e depilar, entre outros), além das vias sexual e vertical (Brasil, 2017; Silva et al., 012). Trata-se de uma doença infecciosa, cujo agente etiológico é um vírus de DNA (Caballero et al., 2018; Yuen et al., 2018), que infecta apenas seres humanos e é uma das principais causas de doença aguda e crônica do fígado. A cronificação da doença coloca os indivíduos em alto risco de morte pelo comprometimento sistêmico e complicações evolutivas como a cirrose e o carcinoma hepatocelular. Em 2015, estima-se que a hepatite B resultou em de 887.000 mortes, principalmente por cirrose e carcinoma hepatocelular (Lindsey \& Hoofnagle, 2008; Organização Mundial da Saúde, 2019; Tsai et al., 2018).

A maneira mais eficaz de reduzir a taxa de mortalidade, complicações evolutivas e a incidência da infecção pelo HBV é por meio dos esquemas de imunização, visto que se diminui a cadeia de transmissão da doença. A vacina contra o HBV é segura e eficaz, já que oferece uma proteção de 98 a 100\% contra a hepatite B (Goel et al., 2016; Hsu et al., 2018; Moghadami et al., 2019; Rodrigues et al., 2019; Tsai et al., 2018;). Além disso, ressalta-se que a vacina contra o HBV também é disponibilizada em formulações combinadas que protegem contra diversas doenças como difteria, poliomielite, tétano, pertussis e Haemophilus influenzae tipo B. Tendo em vista a adesão da população e a redução de custos (Lavanchy, 2012).

Recomenda-se a imunização contra o HBV para pessoas que vivem com HIV (PVHIV) mesmo com a taxa de 
soroproteção alcançada ser subótima, visto que a coinfecção por HIV eleva a concentração de DNA HBV, reduz níveis de enzimas hepáticas e contribui para a progressão de um quadro de cirrose hepática, principalmente em pacientes com redução acentuada dos linfócitos T CD4+ (Neukam et al., 2019; Soriano et al., 2010).

A imunização, diagnóstico e o tratamento antiviral reduziram a incidência de coinfecção por HIV, assim como a carga viral de indivíduos infectados cronicamente (Yuen et al., 2018). No entanto, em algumas regiões do Brasil, de acordo com o Sistema de Informação de Agravos de Notificação (Sinan), entre 2007 e 2018, observa-se uma maior proporção de indivíduos coinfectados, a região Sudeste $(7,8 \%)$ e a Nordeste $(4,4 \%)$. Ademais, um número significativo de indivíduos coinfectados são soropositivos assintomáticos para o vírus da hepatite, ocasionando um diagnóstico tardio e subnotificação (Weissmann et al., 2019).

Esta pesquisa teve como objetivo analisar a cobertura vacinal contra o vírus da hepatite B no estado do Piauí, por meio de um estudo soro epidemiológico de pacientes soropositivos cadastrados no Sistema de Controle de Exames Laboratoriais (SISCEL) do Laboratório Central de Saúde Pública Dr. Costa Alvarenga (LACEN-PI), no ano de 2010.

\section{Metodologia}

A população do estudo foi constituída por 569 indivíduos que vivem com HIV-AIDS, cadastrados no Sistema de Controle de Exames Laboratoriais (SISCEL) do LACEN-PI, em controle de TCD4+/CD8 e carga viral. As variáveis foram escolhidas, considerando as os dados socioeconômicas e os fatores de risco associados a infecção pelo vírus da hepatite B: gênero, idade, raça, escolaridade e marcadores sorológicos para hepatite B, situação conjugal, renda familiar, vacinação contra a hepatite B, contato com suspeito de hepatite B, realizou pequenas cirurgias, realizou cirurgia, realizou transfusão de sangue, fez acupuntura, tem tatuagem, já esteve preso, uso passado ou atual de droga ilícita não endovenosa, uso passado ou atual de droga ilícita endovenosa, preferência sexual e infecções sexualmente transmissíveis (IST).

A determinação do marcador viral para hepatite B Anti-HBs, nas amostras de soro, foi baseada na tecnologia Abbott Laboratories (Abbott) AxSYM $^{\circledR}$ System/MEIA (imunoensaio enzimático por micropartículas) que utiliza o Antígeno de Superfície Recombinante do Vírus da Hepatite B, (rHBsAg) ligado a micropartícula, para determinação quantitativa do anticorpo (anti-HBs).

Os dados foram coletados junto ao banco de dados Sistema de Controle de Exames Laboratoriais (SISCEL) do LACENPI, os achados foram filtrados e organizados em planilha com auxílio dos programas Microsoft Excel® e Statistical Package for the Social Sciences (SPSS 15.0) para comparar as frequências de títulos de anti-HBs entre os indivíduos HIV positivo, usando o nível de significância de 5\% ( $<0,05$ ), com intervalos de 95\% de confiança (IC 95\%), estruturadas nas variáveis com risco biológico de transmissão do vírus da hepatite B.

\section{Resultados e Discussão}

A Tabela 1 mostra a distribuição de indivíduos participantes do estudo de acordo com o sexo e resultado do anti-HBs. No total, 569 pacientes participaram do estudo, sendo 39,9\% do sexo feminino e 60,1\% do sexo masculino. Neste estudo foi observado soropositividade para o anti-HBs em 39,7\% dos participantes, em que 37,4\% dos pacientes do sexo feminino e $41,2 \%$ do sexo masculino apresentaram resultado positivo para o marcador sorológico que está relacionado com a vacinação contra o HBV (anti-HBs $\geq 10 \mathrm{UI} / \mathrm{L})$. 
Tabela 1: Distribuição de pessoas com a presença do anticorpo anti-HBs de acordo o sexo, Teresina, Piauí, 2010.

\begin{tabular}{|c|c|c|c|}
\hline \multicolumn{4}{|c|}{ Anti-HBs } \\
\hline & Positivo & Negativo & Total \\
\hline \multicolumn{4}{|l|}{ Sexo } \\
\hline Masculino & 141 & 201 & 342 \\
\hline Feminino & 85 & 142 & 227 \\
\hline Total & 226 & 343 & 569 \\
\hline
\end{tabular}

Fonte: Autores.

De acordo com os parâmetros socioeconômicos apresentados na Tabela 2, é possível observar que 58,8\% dos pacientes do sexo masculino e 62,5\% do sexo feminino apresentam anti-HBs menor do que $10 \mathrm{UI} / \mathrm{L}$, considerado negativo, sendo a faixa etária entre 31 e 48 anos a que apresenta o maior número de indivíduos com resultados negativos (21,2\%), seguida pela faixa de 18 a $30 \operatorname{anos}(19,5 \%)$.

Tabela 2: Título de anticorpos anti-HBs em indivíduos HIV-infectados de acordo com as variáveis socioeconômicas, Teresina, Piauí, 2010.

\begin{tabular}{|c|c|c|c|c|c|}
\hline \multirow{2}{*}{ Variável } & \multicolumn{2}{|c|}{ Anti-HBs } & \multirow[b]{2}{*}{ Maior que 100} & \multirow[b]{2}{*}{ (a) $\chi^{2}$} & \multirow[b]{2}{*}{ (a) $p$} \\
\hline & Menos de 10 & De 10 a 100 & & & \\
\hline \multicolumn{6}{|l|}{ Sexo } \\
\hline Masculino & 201 & 34 & 107 & \multirow[b]{2}{*}{$1,931^{\mathrm{a}}$} & \multirow[b]{2}{*}{0,378} \\
\hline Feminino & 142 & 26 & 59 & & \\
\hline \multicolumn{6}{|l|}{ Faixa de idade } \\
\hline 18 a 30 & 111 & 11 & 34 & \multirow{4}{*}{$8,099^{\mathrm{a}}$} & \multirow{4}{*}{0,017} \\
\hline 31 a 40 & 121 & 20 & 54 & & \\
\hline 41 a 50 & 72 & 14 & 52 & & \\
\hline$>50$ & 39 & 15 & 26 & & \\
\hline \multicolumn{6}{|l|}{ Raça } \\
\hline Branca & 124 & 27 & 54 & \multirow{5}{*}{$5,284^{\mathrm{a}}$} & \multirow{5}{*}{0,613} \\
\hline Preta & 71 & 12 & 35 & & \\
\hline Parda & 145 & 21 & 77 & & \\
\hline Amarelo & 2 & 0 & 0 & & \\
\hline Indígena & 1 & 0 & 0 & & \\
\hline \multicolumn{6}{|l|}{ Escolaridade } \\
\hline Nenhum & 19 & 0 & 6 & \multirow{4}{*}{$7,638^{\mathrm{a}}$} & \multirow{4}{*}{0,050} \\
\hline $1^{\circ}$ Grau (in)completo & 221 & 41 & 100 & & \\
\hline $2^{\circ}$ Grau (in)completo & 75 & 14 & 38 & & \\
\hline $3^{\circ} \mathrm{Grau}$ (in)completo & 28 & 5 & 22 & & \\
\hline
\end{tabular}




\begin{tabular}{|c|c|c|c|c|c|}
\hline \multicolumn{6}{|l|}{ Situação conjugal } \\
\hline Com Companheiro & 145 & 22 & 63 & \multirow{4}{*}{$4,737^{\mathrm{a}}$} & \multirow[b]{3}{*}{0,705} \\
\hline Sem Companheiro & 144 & 28 & 76 & & \\
\hline Viúvo(a) & 29 & 8 & 14 & & \\
\hline Separado(a) & 19 & 2 & 11 & & \\
\hline Outros & 6 & 0 & 2 & & \\
\hline \multirow{2}{*}{\multicolumn{6}{|c|}{$\begin{array}{l}\text { Renda familiar em } \\
\text { salários mínimos }\end{array}$}} \\
\hline & & & & & \\
\hline Menos de 1 & 127 & 17 & 40 & \multirow{8}{*}{$28,223^{\mathrm{a}}$} & \multirow{8}{*}{0,014} \\
\hline 1 a 2 & 166 & 24 & 90 & & \\
\hline 3 a 4 & 31 & 14 & 24 & & \\
\hline 5 a 6 & 7 & 4 & 8 & & \\
\hline 7 a 10 & 9 & 1 & 1 & & \\
\hline 11 a 20 & 1 & 0 & 2 & & \\
\hline Mais de 20 & 1 & 0 & 0 & & \\
\hline Não sabe & 1 & 0 & 1 & & \\
\hline
\end{tabular}

a) $\chi^{2}$, valor de $p$ e Teste Exato de Fisher quando apropriado. Fonte: Autores.

Quanto à caracterização dos comportamentos que representam fatores de risco para Hepatite B, a Tabela 3 mostra que $50 \%$ dos indivíduos imunizados desenvolveram título de anti-HBs adequado, enquanto os não-imunizados apresentam maior risco pois grande maioria não desenvolve os níveis de anticorpos protetores contra HB acima de 10 UI/L. Dentre os pacientes que realizaram pequenas cirurgias e grandes cirurgias 60,7\% e 59\% apresentaram anti-HBs abaixo de 10 UI/L, respectivamente. Em relação à preferência sexual 61,7\% dos indivíduos heterossexuais e 58,8\% dos indivíduos homossexuais não apresentaram imunização adequada, o que representa um fator de risco para os dois casos.

Tabela 3: Título de anticorpos anti-HBs de acordo com fatores de risco associados à infecção pelo HBV, Teresina, Piauí, 2010.

\begin{tabular}{|c|c|c|c|c|c|}
\hline \multirow{2}{*}{ Variável } & \multicolumn{3}{|c|}{ Anti-HBs } & \multirow{2}{*}{ (a) $\chi^{2}$} & \multirow{2}{*}{ (a) $p$} \\
\hline & Menos de 10 & De 10 a 100 & Maior que 100 & & \\
\hline \multicolumn{6}{|c|}{ Vacinação para } \\
\hline \multicolumn{6}{|c|}{ Hepatite B } \\
\hline \multirow[t]{2}{*}{ Sim } & 136 & 36 & 100 & & \\
\hline & & & & $26,865^{\mathrm{a}}$ & 0,000 \\
\hline Não & 163 & 20 & 44 & & \\
\hline Não Sabe & 44 & 4 & 22 & & \\
\hline
\end{tabular}


Contato com Caso

Suspeito ou

Confirmado de

Hepatite

$\operatorname{Sim}$

Não

Não Sabe

Não se aplica

\section{Pequena Cirurgia}

Sim

Não

\section{Cirurgia}

$\operatorname{Sim}$

Não

Transfusão de

\section{Sangue}

Sim

Não

Acupuntura

Sim

Não

Tatuagem

Sim

Não

309

Não

Uso passado ou

Atual de Droga

Ilícita não

Endovenosa

Sim

Não

160

145

2

130

212

119

221

76

263

Prisão

Sim 37
36

54

11

155

7

53

17

$0,098^{\mathrm{a}}$

0,953

149

$9,822^{\mathrm{a}}$

0,114

58

0

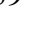

$0,680^{\mathrm{a}}$

0,713

106

62

$0,337^{\mathrm{a}}$

0,845

103

35

$0,353^{\mathrm{a}}$

0,835

127

0,147

1

1

$1,392^{\mathrm{a}}$

0,481 


\begin{tabular}{|c|c|c|c|c|c|}
\hline \multicolumn{6}{|c|}{$\begin{array}{l}\text { Relacionamento } \\
\text { sexual com } \\
\text { usuário de droga }\end{array}$} \\
\hline ilícita & & & & $2,021^{\mathrm{a}}$ & 0,381 \\
\hline Sim & 80 & 18 & 35 & & \\
\hline Não & 224 & 32 & 93 & & \\
\hline \multicolumn{6}{|c|}{ Preferência Sexual } \\
\hline Heterossexual & 282 & 50 & 125 & & \\
\hline Homossexual & 40 & 7 & 21 & $7,799^{\mathrm{a}}$ & 0,264 \\
\hline Bissexual & 20 & 3 & 20 & & \\
\hline $\begin{array}{l}\text { Sem Experiência } \\
\text { Sexual }\end{array}$ & 1 & 0 & 0 & & \\
\hline \multicolumn{6}{|l|}{ IST } \\
\hline Sim & 105 & 28 & 65 & $8,754^{\mathrm{a}}$ & 0,014 \\
\hline Não & 232 & 29 & 98 & & \\
\hline
\end{tabular}

a) $\chi^{2}$, valor de $p$ e Teste Exato de Fisher quando apropriado. Fonte: Autores.

A hepatite B é considerada endêmica em diversos locais do mundo, em especial naqueles em que existem más condições de moradia e higiene. A medida que os países se tornam industrializados é possível observar queda nas taxas de incidência, devido acesso amplo às políticas públicas de saúde, inclusive a vacinação. Estudos mostram que é possível reduzir a hiperendemicidade para baixa, de acordo com os critérios de classificação da OMS (Souto, 2016).

A imunização para a hepatite B no Brasil é realizada por meio de três doses, gratuitas para todos os brasileiros, independentemente de faixa etária no calendário vacinal, de acordo com o Programa Nacional de Imunização (Secretaria de Vigilância em Saúde, 2019). Atualmente, são utilizados testes imunológicos para avaliar se um indivíduo foi vacinado, nesse caso um resultado positivo para anticorpos contra o HBV e negativo para o antígeno do vírus é indicativo de imunização.

Souto (2016) em uma revisão sistemática da epidemiologia do HBV no Brasil no século XXI, observou prevalência entre 55 e $60 \%$ de indivíduos positivos apenas para o anti-HBs, indicativo de cobertura vacinal baixa nas regiões estudadas. Todavia, existe carência de dados na pesquisa referente ao estado do Piauí.

A eficácia protetora da vacina está relacionada à indução de anticorpos anti-HBs e envolve também a indução de células T de memória (Mena, 2015). Entretanto, estudos relatam que as taxas de soroconversão para o anti-HBs em pacientes infectados pelo HIV são atingidas por uma minoria de pacientes tratados (Collier et al., 1988; Fonseca et al., 2005; Hadziyannis et al., 2003; Soriano et al., 2005; Konopnick et al., 2005), sendo a imunodeficiência o principal fator atribuído aos baixos índices de soroconversão, uma vez que pacientes não infectados pelo HIV apresentam resposta adequada com taxas de soroconversão atingindo até 94\% (Carne et al., 1987; Collier et al., 1988; Keet et al., 1992). No presente estudo os valores de soroconversão para o anti-HBs foram observados em apenas 39,7\% dos participantes, o que confirma que ocorre menores taxas da produção desses anticorpos em pacientes imunocomprometidos pelo HIV.

Devido às formas de transmissão do vírus da hepatite B serem as mesmas do HIV, a coinfecção é comum, condição em que a patogenicidade do HBV se torna maior, podendo acelerar danos ao fígado. Por ser uma morbidade grave e que demanda altos gastos do sistema de saúde, é imprescindível expandir a vacinação de pacientes soropositivos, mesmo que alguns estudos mostrem que existe resposta prejudicada frente à imunização (Martins et. al, 2015). Nesse mesmo estudo, que avaliou a cobertura 
vacinal de pacientes HIV-positivos no Sul do Brasil, foi observada cobertura de 57,4\% da população estudada, dentre estes, 47\% apresentou anti-HBs maior que $10 \mathrm{UI} / \mathrm{L}$.

Tonial e colaboradores (2011) estudou os marcadores sorológicos em estudantes do ensino fundamental, entre 10 e 15 anos de idade, observando baixa taxa de infecção, que pode ser atribuída à diversos fatores que reduzem o risco de contaminação, podendo-se destacar a baixa exposição decorrente do uso de drogas injetáveis, uma das principais formas de transmissão entre adultos. A taxa de cobertura vacinal observada foi de $97,5 \%$, sendo observado que 51,8\% dos participantes obtiveram valores de anti-HBs maior do que $10 \mathrm{UI} / \mathrm{L}$ isoladamente.

Trabalhadores da saúde estão expostos diariamente a situações que aumentam a probabilidade de adquirir diversas doenças, inclusive hepatite B, dessa forma, faz-se de fundamental importância proteger essas pessoas para reduzir as taxas de incidência, sendo a vacinação a principal medida preventiva. Em estudo realizado por Souza et al. (2015) a prevalência de vacinação para o HBV foi de 59,9\% entre os trabalhadores de saúde, considerada baixa quando comparada com outros estudos.

A cobertura vacinal em pessoas que vivem com HIV observada neste estudo foi menor do que a taxa indicada no Departamento de Informática do SUS para a população em geral do estado do Piauí para a época da pesquisa (95,7\% no ano de 2010), estando abaixo do encontrado por Martins et al. (2015) em pacientes soropositivos.

Observa-se que a maioria do público imunizado contra o HBV desenvolve uma resposta protetora efetiva com alta concentração de anticorpos protetores, entretanto, existem algumas condições que podem reduzir a resposta imune em indivíduos e, consequentemente, esses indivíduos continuam suscetíveis à infecção pelo HVB, dentre esses fatores pode-se destacar o tabagismo, obesidade, idade maior que 40 anos e imunodeficiência (Koc et al., 2019). Na imunodeficiência decorrente da Síndrome da Imunodeficiência Humana (AIDS) causada pelo Vírus da Imunodeficiência Humana (HIV) ocorre a redução de linfócitos B afetando a manutenção da proteção a longo prazo contra o HBV devido a menor produção de imunoglobulinas (Mena et al., 2015).

Como limitações, o presente estudo está sujeito a viés de seleção e de resposta, tendo em vista que as questões apresentadas em algum momento pode não ter sido compreendida ou pode ter causado algum temos de serem repreendidos devido a sua natureza. Como medida de tentar minimizar estas limitações, a pesquisa foi conduzida com número bastante representativo da população HIV-AIDS e o questionário foi apresentado de forma tranquiila pelo pesquisador, aguardando as respostas sem açodamento.

\section{Conclusão}

Os resultados apontam para um déficit de vacinação em pessoas que vivem com HIV no Estado do Piauí, visto que o número de pacientes vacinados nesta pesquisa é baixo, assim como os dados de soroconversão apontam para reduzida taxa de imunização efetiva deste grupo. De acordo com os títulos de anti-HBs foram observados comportamentos-chave de vulnerabilidade que torna os pacientes suscetíveis à infecção, destacando-se: a renda familiar e escolaridade, que apontam para a necessidade de implementação de políticas públicas que atendam a população menos favorecida.

\section{Referências}

Brasil. Ministério da Saúde. Secretaria de Vigilância em Saúde. Departamento de DST, Aids e Hepatites Virais. Protocolo Clínico e Diretrizes Terapêuticas para Hepatite B e Coinfecções/Ministério da Saúde, Secretaria de Vigilância em Saúde, Departamento de DST, Aids e Hepatites Virais. Ministério da Saúde, 2017.

Caballero, A., Tabernero, D., Buti, M., \& Rodriguez-Frias, F. (2018). Hepatitis B virus: The challenge of an ancient virus with multiple faces and a remarkable replication strategy. Antiviral research, 158, 34-44.

Carne, C. A., Weller, I. V., Waite, J., Briggs, M., Pearce, F., Adler, M. W., \& Tedder, R. S. (1987). Impaired responsiveness of homosexual men with HIV antibodies to plasma derived hepatitis B vaccine. Br Med J (Clin Res Ed), 294(6576), 866-868.

Collier, A. C., Corey, L., Murphy, V. L., \& Handsfield, H. H. (1988). Antibody to human immunodeficiency virus (HIV) and suboptimal response to hepatitis $\mathrm{B}$ vaccination. Annals of internal medicine, 109(2), 101-105. 
Silva, A. L., Vitorino, R. R., Esperidião-Antonio, V., Santos, E. T., Santana, L. A., Henriques, B. D., \& Gomes, A. P. (2012). Hepatites virais: B, C e D: atualização. Rev Bras Clin Med, 10(3), 206-18.

Davitkov, P., \& Falck-Ytter, Y. (2019). Reactivation of Hepatitis B. In Liver Disease (pp. 279-289).

Souza, F. O., Freitas, P. S. P., Araújo, T. M., \& Gomes, M. R. (2015). Vacinação contra hepatite B e Anti-HBS entre trabalhadores da saúde. Cadernos Saúde Coletiva, 23 (2): 172-179.

Fonseca, M. O., Pang, L. W., Paula Cavalheiro, N., Barone, A. A., \& Lopes, M. H. (2005). Randomized trial of recombinant hepatitis B vaccine in HIV-infected adult patients comparing a standard dose to a double dose. Vaccine, 23(22), 2902-2908.

Goel, A., Aggarwal, A., \& Aggarwal, R. (2016). Hepatitis B vaccine: Using skin when muscle does not work.

Hadziyannis, S. J., Tassopoulos, N. C., Heathcote, E. J., Chang, T. T., Kitis, G., Rizzetto, M., \& Xiong, S. (2003). Adefovir dipivoxil for the treatment of hepatitis B e antigen-negative chronic hepatitis B. New England Journal of Medicine, 348(9), 800-807.

Organização Mundial da Saúde. (2019). Hepatitis B. Acessado em 26 Novembro 2019, em https://www.who.int/news-room/fact-sheets/detail/hepatitis-b

Hsu, Y. C., Ho, H. J., Lee, T. Y., Huang, Y. T., Wu, M. S., Lin, J. T., \& El-Serag, H. B. (2018). Temporal trend and risk determinants of hepatocellular carcinoma in chronic hepatitis B patients on entecavir or tenofovir. Journal of viral hepatitis, 25(5), 543-551.

Keet, I. P., Van Doornum, G., Safary, A., \& Coutinho, R. A. (1992). Insufficient response to hepatitis B vaccination in HIV-positive homosexual men. AIDS (London, England), 6(5), 509.

Koc, Ö. M., Menart, C., Theodore, J., Kremer, C., Hens, N., Koek, G. H., \& Oude Lashof, A. M. (2019). Ethnicity and response to primary three-dose hepatitis B vaccination in employees in the Netherlands, 1983 through 2017. Journal of Medical Virology.

Lavanchy, D. (2012). Viral hepatitis: global goals for vaccination. Journal of Clinical Virology, 55(4), 296-302.

Martins, S., Livramento, A. D., Andrigueti, M., Kretzer, I. F., Machado, M. J., Spada, C., \& Treitinger, A. (2015). Vaccination coverage and immunity against hepatitis B among HIV-infected patients in South Brazil. Brazilian Journal of Infectious Diseases, 19(2), 181-186.

Mena, G., García-Basteiro, A. L., \& Bayas, J. M. (2015). Hepatitis B and A vaccination in HIV-infected adults: A review. Human vaccines \& immunotherapeutics, 11(11), 2582-2598.

Moghadami, M., Dadashpour, N., Mokhtari, A. M., Ebrahimi, M., \& Mirahmadizadeh, A. (2019). The effectiveness of the national hepatitis B vaccination program 25 years after its introduction in Iran: a historical cohort study. The Brazilian Journal of Infectious Diseases.

Neukam, K., Gutiérrez-Valencia, A., Llaves-Flores, S., Espinosa, N., Viciana, P., \& López-Cortés, L. F. (2019). Response to a reinforced hepatitis B vaccination scheme in HIV-infected patients under real-life conditions. Vaccine, 37(20), 2758-2763.

Rodrigues, I. C., Silva, R. D. C. M. A., Felício, H. C. C. D., \& Silva, R. F. D. (2019). New immunization schedule effectiveness against hepatitis B in liver transplantation patients. Arquivos de gastroenterologia, 56(4), 440-446.

Soriano, V., Mocroft, A., Peters, L., Rockstroh, J., Antunes, F., Kirkby, N., \& Lundgren, J. (2010). Predictors of hepatitis B virus genotype and viraemia in HIVinfected patients with chronic hepatitis B in Europe. Journal of antimicrobial chemotherapy, 65(3), 548-555.

Souto, F. J. D. (2016). Distribution of hepatitis B infection in Brazil: the epidemiological situation at the beginning of the 21 st century. Revista da Sociedade Brasileira de Medicina Tropical, 49(1), 11-23.

Tonial, G. C., Passos, A. M., Livramento, A. D., Scaraveli, N. G., Batschauer, A. P. D. B., Bueno, E. C., \& Treitinger, A. (2011). Hepatitis B marker seroprevalence and vaccination coverage in adolescents in the City of Itajaí, State of Santa Catarina, Southern Brazil, in 2008. Revista da Sociedade Brasileira de Medicina Tropical, 44(4), 416-419.

Tsai, K. N., Kuo, C. F., \& Ou, J. H. J. (2018). Mechanisms of hepatitis B virus persistence. Trends in microbiology, 26(1), 33-42.

Weissmann, L., de Melo Picone, C., Gouvêa, M. S. G., Ferreira, P. R. A., Viana, M. S. V. B., Pinho, J. R. R., \& Segurado, A. C. (2019). Hepatitis B viremia in HIV-coinfected individuals under antiretroviral therapy. The Brazilian Journal of Infectious Diseases.

World Health Organization. (2017). Global hepatitis report 2017. World Health Organization.

Yuen, M. F., Chen, D. S., Dusheiko, G. M., Janssen, H. L., Lau, D. T., Locarnini, S. A., \& Lai, C. L. (2018). Hepatitis B virus infection. Nature Reviews Disease Primers, 4, 18035. 